\title{
Association of Anticardiolipin Antibodies and Extent of Coronary Artery Disease in Military Personnel and Non Military Population With Acute Coronary Syndrome
}

\author{
Bahareh Hajibaratali ${ }^{1,}$, Shahram Baharvand ${ }^{2}$, Shahrooz \\ Yazdani $^{3}$ \\ ${ }^{1}$ Department of Cardiology, Labafinejad Hospital, Shahid Beheshti University of \\ Medical Sciences, Tehran, Iran \\ ${ }^{2}$ Department of Rheumatolog, Aja University of Medical Sciences, Tehran, Iran \\ ${ }^{3}$ Department of Cardiology, Shahid Rajaei Hospital Medical Center, Alborz \\ University of Medical Sciences, Tehran, Iran \\ * Corresponding author: Bahareh Hajibaratali, Department of Cardiology, La- \\ bafinejad Hospital, Shahid Beheshti University of Medical Sciences, Tehran, Iran. \\ E-mail: baharehbarati@yahoo.com
}

DOI: $10.21859 / \mathrm{ijcp}-010306$

\begin{tabular}{l} 
Submited: 02.19 .2016 \\
Accepted: 08.10 .2016 \\
\hline
\end{tabular}

Keywords:

Antibodies Anticardiolipin

Coronary Artery Disease

Angiography

Military Personnel

(C) 2016. International Journal of Cardiovascular Practice.

\begin{abstract}
Introduction: Cardiac involvement is one of the chief complications considerably contributing to the morbidity and mortality of patients with systemic autoimmune diseases. Anticardiolipin antibody is a marker of elevated myocardial infarction risk and it also predicts post cardiac intervention risk. In the current study, we aimed to evaluate the association between anticardiolipin $\operatorname{IgM}$ and $\operatorname{IgG}$ levels and atherosclerotic involvement of coronary arteries.

Methods: Patients with acute coronary syndrome admitted to a military hospital were included in the study. Patients were categorized to military personnel and non-military personnel. Laboratory data including lipid profile, blood sugar, anticardiolipin IgM and anticardiolipin IgG were verified. Existence and the extent of Coronary Artery Disease (CAD) were defined according to angiographic findings. The relationship between anticardiolipin antibody levels and the number of vessels were evaluated.

Results: According to our sample population calculation, we performed the study on a total of 92 patients. Measurement of both anticardiolipin antibodies (IgM and $\operatorname{IgG}$ ) in military personnel and non personnel patients showed no significant difference. In both military personnel and non personnel groups, there was a significant association between anticardiolipin IgM and IgG levels and number of coronary arteries with significant stenosis. The C Reactive Protein (CRP) level was significantly higher in military personnel. Conclusions: According to the study results, anticardiolipin antibody levels were the same in both military personnel and non personnel. Also systolic and diastolic blood pressures were not significantly different in both groups. Increased CRP level in military personnel may be a warning signal about the possibility of premature CAD in this population, hence aggressive risk factor modification is recommended. Paradoxically lipid profile and FBS levels were more favorable in military personnel, which indirectly reflects their higher state of physical activity.
\end{abstract}

\section{INTRODUCTION}

Atherosclerosis is a multifactorial process, which initiates in early life but presents clinically later in life. Atherosclerosis is progressively more considered an immune-mediated process. Epidemiological studies revealed an increase of cardiovascular events in patients affected by systemic autoimmune diseases. All the anatomical heart structures can be affected, and several pathogenic mechanisms have been reported [1]. Antiphospholipid Syndrome (APS) is a disorder that man- ifests clinically as recurrent venous or arterial thrombosis and/or fetal loss. Antiphospholipid (APL) antibodies are a group of antibodies directed against epitopes on plasma proteins that are uncovered by binding of these proteins to anionic phospholipids on plasma membranes. Characteristic laboratory abnormalities in APS, include persistently elevated levels of antibodies directed against membrane anionic phospholipids (i.e. Anticardiolipin [ACL] antibody, 
antiphosphatidylserine) or their associated plasma proteins, predominantly beta- 2 glycoprotein I (apolipoprotein $\mathrm{H}$ ), or evidence of a circulating anticoagulant [2-5].

Anticardiolipin antibody is a member of autoantibodies, which directly acts against protein- phospholipid complex. Elevated levels of these antibodies are associated with a clinical syndrome in which venous and arterial thrombosis occurs [6-9]. In addition to antiphospholipid antibody syndrome, anticardiolipin level elevates in a variety of autoimmune diseases especially in SLE [10]. A subset of people with no apparent autoimmune disease, have elevated anticardiolipin levels.

Early findings about the clinical importance of anticardiolipin antibodies came from SLE patients [11]. In SLE, patients with higher titers of anticardiolipin antibody had more vascular events including arterial thrombosis [12]. Interestingly, $14 \%$ of patients with thrombotic brain ischemia had marked elevation of anticardiolipin antibody $[13,14]$. Anticardiolipin antibody is a marker of elevated myocardial infarction risk and it also predicts post cardiac intervention risk [15].

These observations support the possible role of autoimmunity in the genesis of atherosclerosis that may have clinical or subclinical features. The clinical edge of this phenomenon is Coronary Artery Diseases (CAD), while early endothelial dysfunction, abnormalities of circulation or atherosclerotic plaques, detected by different imaging techniques, identifies the subclinical atherosclerosis expression. According to different reports, traditional risk factors were not different in APS and in the general population [16]. In agreement with these findings, APS patients have an increased rate of cardiovascular incidents: myocardial infarction appears at same stage of the disease in up to $5.5 \%$ and is the presenting manifestation in $2.8 \%$ of APS patients [17].

In spite of the mentioned studies, some researchers have shown that there is no robust association between anticardiolipin levels and cardiovascular risk. In addition, evidence for the relationship between anticardiolipin antibody and atherosclerosis is limited.In the current study, we aimed to evaluate the association between anticardiolipin IgM and IgG levels and atherosclerotic involvement of coronary arteries with an angiographic study.

\section{METHODS}

This study had a cross sectional design in order to evaluate the relationship between anticardiolipin level and the extent of coronary artery disease. Patients with acute coronary syndrome admitted to 502 military hospitals of the Islamic Republic of Iran were included in the study. Patients were categorized to two groups. The first group comprised of military personnel, who were admitted with Acute Coronary Syndrome (ACS) diagnosis and underwent coronary angiography to delineate the coronary anatomy, this group was defined as our case group. The second group composed of non military patients, who were admitted to the 502 military hospitals with the diagnosis of ACS and underwent coronary angiography, this group was defined as the control group. All patients with ACS and the high risk criteria became candidates of coronary angiography, according to our current standard guidelines. Exclusion criteria included any active or chronic inflammatory disease, acute or chronic infectious disease, known autoimmune disease and consumption of certain drugs including phenothiazin, hydralazine, procainamide and prednisone within the past year. To determine the sample population we relied on data obtained by the study of Eber et al [18].

Demographic, clinical and paraclinical data were gathered in a questionnaire, according to the medical records of the patients. Clinical variables including systolic and diastolic blood pressures were recorded. Laboratory data including lipid profile, blood sugar, anticardiolipin IgM and anticardiolipin IgG were verified. Existence and the extent of CAD was defined according to the angiographic findings. Significant CAD was defined as the existence of more than $50 \%$ diameter luminal stenosis of the coronary arteries. Once concent was provided by the patients, $5 \mathrm{cc}$ of blood was drained extra to the routine blood sampling to measure the anticardiolipin level with the Enzyme Linked Immunosorbent Assay (ELISA). The study protocol was approved by the medical ethics committee of the Islamic Republic of Iran military force and all procedures were designed according to the declaration of Helsinki. After providing detailed oral information to participants, written informed consent was obtained from each participant. The study was intended to be triple blind. Patients, laboratory technicians, physicians in charge of angiography and the statistics specialist were all unaware of the aims of study and about the importance of comparison between military personnel and non personnel patients.

To assess variables, quantitative results were declared in the form of mean +/- standard deviation and qualitative variables were stated with their percentages. In order to evaluate the relationship between the anticardiolipin antibody levels ( $\operatorname{IgM}$ and $\operatorname{IgG}$ ) and the number of vessels with significant stenosis in angiography, we used One-way Analysis of Variance (ANOVA) statistical test.

In order to document the association between employment in the military service and anticardiolipin antibody level and number of involved coronary arteries, we used the Cumulative Logit Modeling statistical test. To analyze the results, the SPSS software version 9.1 and SAS software version 16 were used. For stating the statistical significance, $\mathrm{p}$ values of level less than 0.05 were considered statistically meaningful.

\section{RESULTS}

According to our sample population calculation, we performed the study on a total of 92 patients (46 patients in military personnel group and 46 patients in the nonmilitary personnel group). All patients in the military personnel group were male while only $58.7 \%$ of participants in the non military group were male. Patients in the military personnel group were significantly younger than the patients in the non-military personnel group. There was no significant difference in systolic blood pressure in the two groups (Table 1).

In the laboratory findings, $C$ Reactive Protein (CRP) level was significantly higher in the military personnel group than the non-military personnel. In contrast, military personnel had significantly lower total cholesterol and Fasting Blood Sugar (FBS) levels and higher high density cholesterol level (Table 1). Furthermore, $60.9 \%$ of military personnel and $80.3 \%$ of non military personnel had no significant stenosis of coronary arteries in angiography. Distribution of involved vessels and the frequency of left main disease were the same in the two groups but the percentage of three vessel disease was higher in the military personnel than the non personnel (19.6\% versus $10.9 \%$ ) (Table 2 ). 
Table 1 Demography and Baseline Laboratory Finding of the Patients

\begin{tabular}{|lccc|}
\hline Features & Military Personnel & Non Personnel & P value \\
Male & $46(100)$ & $27(58.7)$ & $<0.001$ \\
Age (years) & $49.6 \pm 5.6$ & $54.4 \pm 11.1$ & 0.01 \\
Systolic blood pressure (mmHg) & $132.2 \pm 17.2$ & $126.0 \pm 17.8$ & 0.127 \\
Positive CRP & $12(21.7)$ & $1(2.2)$ & 0.004 \\
ESR (mm/h) & $14.3 \pm 9.1$ & $16.9 \pm 14$ & 0.327 \\
FBS (mg/dL) & $102.7 \pm 23.4$ & $139.4 \pm 20.5$ & $<0.001$ \\
Total cholesterol (mg/dL) & $234.9 \pm 59.2$ & $265.9 \pm 42.5$ & 0.006 \\
Triglyceride (mg/dL) & $158.3 \pm 35.6$ & $161.5 \pm 79.8$ & 0.810 \\
LDL (mg/dL) & $119.6 \pm 29.3$ & $129.7 \pm 39.4$ & 0.187
\end{tabular}

CRP: C reactive protein; ESR: erythrocyte sedimentation rate; FBS: fasting blood sugar; HDL: high density cholesterol; LDL: low density cholesterol

Data are presented as No (\%) or mean \pm SD

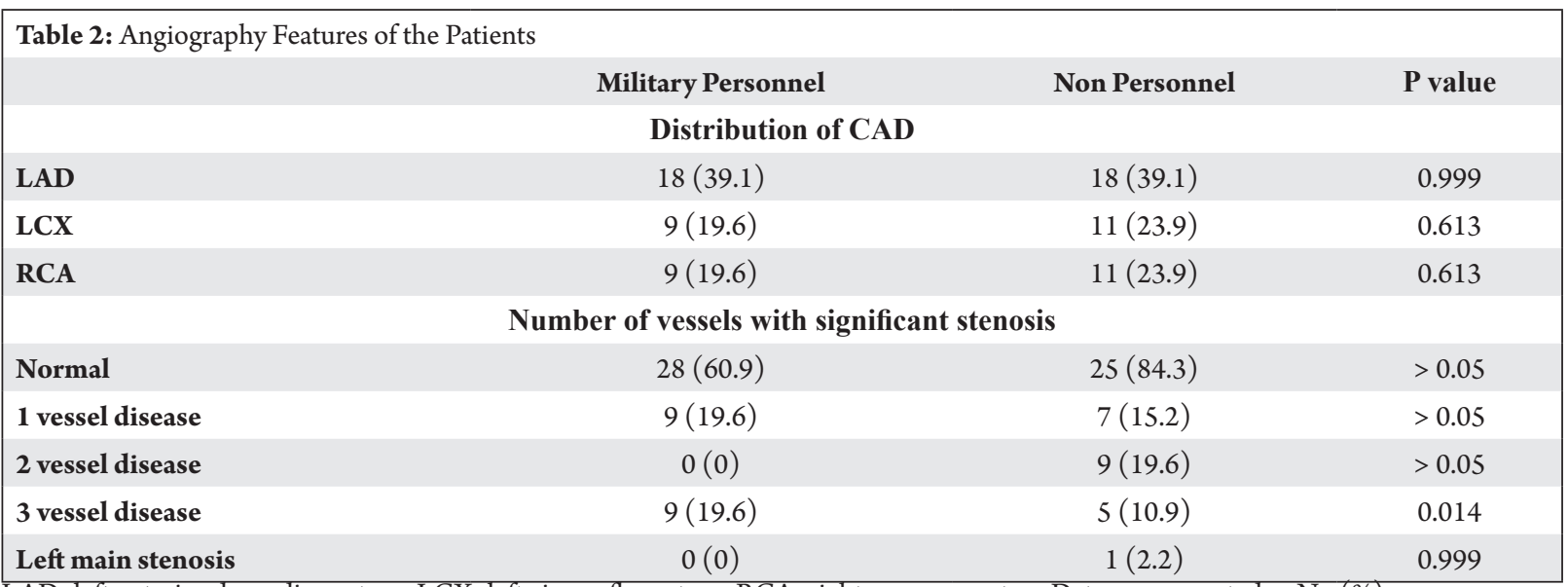

LAD: left anterior desending artery; LCX: left circumflex artery; RCA: right coronary artery Data are presented as No (\%)

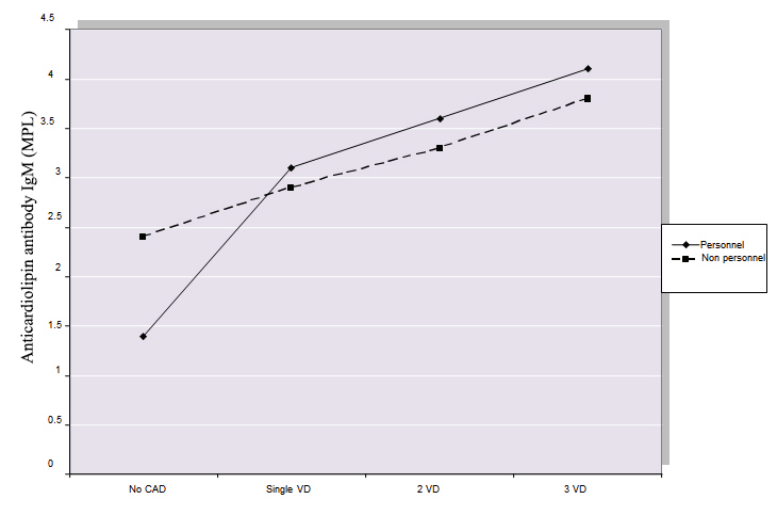

Figure 1: Association of Anticardiolipin Antibody IgM and Number of Coronary Stenosis

Measurement of both anticardiolipin antibodies (IgM and $\operatorname{IgG}$ ) in military personnel and non personnel patients showed no significant difference. Consequently, employment in the military service does not alter the level of anti- cardiolipin antibodies. Anticardiolipin IgM antibody level in military personnel and military non personnel patients were 1.973.04 \pm MPL units and 1.94 \pm 2.66 MPL units, respectively. Anticardiolipin IgG antibody level in military personnel and military non personnel were $2.55 \pm 4.14 \mathrm{GPL}$ units and $2.71 \pm 3.88$ GPL units, respectively. In both military personnel and non personnel groups, there was a significant association between anticardiolipin IgM levels and number of coronary arteries with significant stenosis (Fig 1).

Anticardiolipin antibody IgM level in patients with normal coronary arteries in military personnel and non military personnel were 1.4 and 2.4 MPL units, respectively. The IgM antibody level with single vessel coronary artery disease in military personnel and non military personnel were 3.1 and 2.9 MPL units, respectively. In patients with two vessel coronary artery disease, IgM antibody level in military personnel and military non personnel patients were respectively 3.6 and 3.3 MPL units. Finally three vessel coronary artery involvement in military personnel and non personnel groups had IgM antibody level of 4.1 and 3.8 MPL units, respectively $(\mathrm{P}<0.05)$. In addition, in both military personnel and non personnel groups, there was a significant association between 
anticardiolipin IgG levels and number of coronary arteries with significant stenosis (Table 3).

\begin{tabular}{|lccc|}
\hline Table 3: Anticardiolipin IgG Antibody Level in Patients Who Underwent Angiography & & \\
IgG level (GPL) & Military Personnel & Non Personnel & P value \\
Normal Coronary arteries & $1.5 \pm 0.1$ & $3.2 \pm 0.2$ & $<0.05$ \\
1 vessel disease & $3 \pm 0.2$ & $3.6 \pm 0.3$ & $<0.05$ \\
2 vessel disease & $4.1 \pm 0.5$ & $3.9 \pm 0.3$ & $<0.05$ \\
3 vessel disease & $5.4 \pm 0.3$ & $4.9 \pm 0.5$ & $<0.05$ \\
\hline
\end{tabular}

Data are presented as mean \pm SD

\section{DISCUSSION}

Some novel risk factors that could contribute to atherosclerosis development have been reported recently. These include inflammatory markers, including $\mathrm{CRP}$, fibrinogen, interleukin-6, CD40/CD40L, adhesion molecules, and immunological factors including anticardiolipin (ACL), anti-ß2GPI, anti-oxLDL and anti-HSP antibodies. Several autoantibodies are associated with atherosclerosis and its manifestations in humans [19].

Antiphospholipid Syndrome is a prothrombotic state characterized by recurrent arterial and venous thrombosis, recurrent pregnancy loss, and the presence of circulating antiphospholipid antibodies. Thrombophilia may be associated with premature atherosclerosis, and accelerated atherosclerosis was suggested as an additional clinical feature of APS. This pathological process may be mediated by direct proinflammatory and procoagulant activity that APLs exert on endothelial cells or indirectly, via the inflammatory/immune mechanisms that have been implicated in autoantibody-mediated thrombosis [20]. Veres et al. showed a correlation between serum levels of ACL and anti- $\beta 2$ GPI antibodies and the incidence and severity of acute coronary syndrome, MI and stroke [21]. Therefore, non-traditional risk factors such as antibodies seem to be involved in APS-associated atherogenesis. In vitro, APL accelerates the process of plaques formation, enhancing the macrophages transformation to foam cells by oxLDL [22].

The actual frequency of APS in the general population is unknown. One to $5 \%$ of healthy individuals have APL antibodies. Furthermore, ACL antibodies tend to be found more frequently in elderly persons; thus, positive titer results should be interpreted with caution in this population. The APL antibodies are found in approximately $30-40 \%$ of patients with SLE, but only about $10 \%$ have APS [23].

Antiphospholipid Syndrome is more common in young to middle-aged adults; however, it also manifests in children and elderly people. A female predominance has been documented, particularly for secondary APS. This parallels the association of APS with SLE and other connective-tissue diseases, which also have a female predominance [24]. Patients with APS are prone to cardiovascular events. Coronary artery events and valvular heart disease are common features of this syndrome [25]. Consequently, early diagnosis, aggressive risk factor modification and proper management may reduce the cardiovascular burden in patients with APS. In this study, in addition to the measurement of anticardiolipin antibody levels and evaluation of their relationship with number of coronary artery stenosis, we further explored the differences in military and nonmilitary personnel.

According to the study results, anticardiolipin antibody levels were the same in both military personnel and non personnel patients. Also systolic and diastolic blood pressures were not significantly different in both groups. As mentioned in the result section, CRP level was higher in the military personnel group. Chronic inflammation is pivotal in heart disease; studies have shown that high levels of CRP, measured by high-sensitivity CRP (hs-CRP), can be a marker of atherosclerosis. High sensitivity-CRP is an important predictor for cardiovascular events, including myocardial infarction, cerebrovascular events, peripheral vascular disease and sudden cardiac death in individuals without a history of heart disease. In patients with acute coronary disease, CRP level predicts mortality and cardiac complications [26]. High CRP levels portend a worse prognosis in patients with acute coronary syndromes. High sensitivity-CRP is also a marker of metabolic syndrome [27].

Increased CRP level in military personnel may be a warning signal about the possibility of premature $\mathrm{CAD}$ in this population, hence aggressive risk factor modification is recommended. Paradoxically, lipid profile and FBS levels were more favorable in military personnel group, which indirectly reflects their higher state of physical activity. In angiography, as stated although military personnel had higher frequency of coronary artery disease, the difference was not statistically significant. The subgroup of patients with three-vessel CAD had significantly higher frequency in military personnel. Considering the higher CRP level in military personnel, and the predictive value of $\mathrm{CRP}$, aggressive modification of CRP and inflammatory state in all patients and particularly in military personnel seems reasonable [28-31].

According to the findings of the current article, anticardiolipin antibody levels are not different in military and non military personnel but in both groups the higher titers of antibodies are associated with more extensive CAD. In conclusion, in this study we showed the importance and association of anticardiolipin antibodies and CRP with the extent of CAD. Further research is recommended for exploring these issues to target the atherosclerosis process more efficiently.

\section{REFERENCES}

1. Wallace DJ, Hahn B. Dubois' Lupus Erythematosus. Philadelphia: Lippincott Williams \& Wilkin; 2007.

2. Amengual O, Atsumi T, Koike T. Pathophysiology of thrombosis and potential targeted therapies in antiphospholipid syndrome. Curr Vasc Pharmacol. 2011;9(5):606-18. PMID: 21692741

3. Lim W. Complement and the antiphospholipid syndrome. Curr Opin Hematol. 2011;18(5):361-5. DOI: $10.1097 / \mathrm{MOH} .0 \mathrm{~b} 013 \mathrm{e}-$ 3283497f3e PMID: 21730831

4. Passam FH, Giannakopoulos B, Mirarabshahi P, Krilis SA. Molecular pathophysiology of the antiphospholipid syndrome: the role of oxidative post-translational modification of beta 2 glycoprotein I. J Thromb Haemost. 2011;9 Suppl 1:275-82. DOI: 10.1111/j.15387836.2011.04301.x PMID: 21781264 
5. Sangle NA, Smock KJ. Antiphospholipid antibody syndrome. Arch Pathol Lab Med. 2011;135(9):1092-6. DOI: 10.5858/2010-0325RSR.1 PMID: 21877992

6. Wilson WA, Gharavi AE, Koike T, Lockshin MD, Branch DW, Piette $\mathrm{JC}$, et al. International consensus statement on preliminary classification criteria for definite antiphospholipid syndrome: report of an international workshop. Arthritis Rheum. 1999;42(7):1309-11. DOI: 10.1002/1529-0131(199907)42:7<1309::AID-ANR1>3.0.CO;2-F PMID: 10403256

7. Hughes G. The antiphospholipid syndrome: ten years onLancet: Gulf Professional Publishing; 1993.

8. Brenner B, Blumenfeld Z, Markiewicz W, Reisner SA. Cardiac involvement in patients with primary antiphospholipid syndrome. J Am Coll Cardiol. 1991;18(4):931-6. DOI: 10.1016/0735$\underline{1097(91) 90749-y}$

9. Lechner K, Eichinger S, Jäger U, Pabinger I, Kyrle PA. Lupus anticoagulant and clinical correlates. Thromb Res. 1992;65:S1. DOI: 10.1016/0049-3848(92)90320-a

10. Zuckerman E, Toubi E, Shiran A, Sabo E, Shmuel Z, Golan TD, et al. Anticardiolipin antibodies and acute myocardial infarction in non-systemic lupus erythmatosus patients: a controlled prospective study. Am J Med. 1996;101(4):381-6. DOI: 10.1016/S00029343(96)00226-4 PMID: 8873508

11. Anticardiolipin antibodies are an independent risk factor for first ischemic stroke. The Antiphospholipid Antibodies in Stroke Study (APASS) Group. Neurology. 1993;43(10):2069-73. PMID: $\underline{8413969}$

12. Babikian VL, Brey RL, Coull BM, Dewitt LD, Feinberg WM, Feldmann E, et al. Clinical and laboratory findings in patients with antiphospholipid antibodies and cerebral ischemia. Stroke. 1990;21(9):1268-73.

13. Hess DC, Krauss J, Adams RJ, Nichols FT, Zhang D, Rountree HA. Anticardiolipin antibodies: a study of frequency in TIA and stroke. Neurology. 1991;41(4):525-8. PMID: 2011251

14. Trimble M, Bell DA, Brien W, Hachinski V, O’Keefe B, McLay C, et al. The antiphospholipid syndrome: Prevalence among patients with stroke and transient ischemic attacks. Am J Med. 1990;88(6):593-7. DOI: $10.1016 / 0002-9343(90) 90523-g$

15. Phadke KV, Phillips RA, Clarke DT, Jones M, Naish P, Carson P. Anticardiolipin antibodies in ischaemic heart disease: marker or myth? Br Heart J. 1993;69(5):391-4. PMID: 8518060

16. Shoenfeld Y, Gerli R, Doria A, Matsuura E, Cerinic MM, Ronda N, et al. Accelerated atherosclerosis in autoimmune rheumatic diseases. Circulation. 2005;112(21):3337-47. DOI: 10.1161/CIRCULATIONAHA.104.507996 PMID: 16301360

17. Cervera R, Piette JC, Font J, Khamashta MA, Shoenfeld Y, Camps MT, et al. Antiphospholipid syndrome: clinical and immunologic manifestations and patterns of disease expression in a cohort of 1,000 patients. Arthritis Rheum. 2002;46(4):1019-27. PMID: 11953980

18. Eber B, Schumacher M, Auer-Grumbach P, Toplak H, Klein W. In- creased IgM-anticardiolipin antibodies in patients with restenosis after percutaneous transluminal coronary angioplasty. Am J Cardiol. 1992;69(16):1255-8. PMID: 1585855

19. George J, Afek A, Gilburd B, Levy Y, Blank M, Kopolovic J, et al. Atherosclerosis in LDL-receptor knockout mice is accelerated by immunization with anticardiolipin antibodies. Lupus. 1997;6(9):723-9. PMID: 9412988

20. Shoenfeld Y, Harats D, George J. Atherosclerosis and the antiphospholipid syndrome: a link unravelled? Lupus. 1998;7 Suppl 2:S1403. PMID: 9814692

21. Veres K, Lakos G, Kerenyi A, Szekanecz Z, Szegedi G, Shoenfeld Y, et al. Antiphospholipid antibodies in acute coronary syndrome. Lupus. 2004;13(6):423-7. PMID: 15303568

22. Delgado Alves J, Ames PR, Donohue S, Stanyer L, Nourooz-Zadeh J, Ravirajan C, et al. Antibodies to high-density lipoprotein and beta2-glycoprotein I are inversely correlated with paraoxonase activity in systemic lupus erythematosus and primary antiphospholipid syndrome. Arthritis Rheum. 2002;46(10):2686-94. DOI: $10.1002 /$ art.10542 PMID: 12384928

23. Lockshin MD. Update on antiphospholipid syndrome. Bull NYU Hosp Jt Dis. 2006;64(1-2):57-9. PMID: 17121491

24. Avcin T, Cimaz R, Silverman ED, Cervera R, Gattorno M, Garay $\mathrm{S}$, et al. Pediatric antiphospholipid syndrome: clinical and immunologic features of 121 patients in an international registry. Pediatrics. 2008;122(5):e1100-7. DOI: 10.1542/peds.2008-1209 PMID: $\underline{18955411}$

25. Koniari I, Siminelakis SN, Baikoussis NG, Papadopoulos G, Goudevenos J, Apostolakis E. Antiphospholipid syndrome; its implication in cardiovascular diseases: a review.J Cardiothorac Surg. 2010;5:101. DOI: $10.1186 / 1749-8090-5-101$ PMID: 21047408

26. Casas JP, Shah T, Hingorani AD, Danesh J, Pepys MB. C-reactive protein and coronary heart disease: a critical review. J Intern Med. 2008;264(4):295-314. DOI: 10.1111/j.1365-2796.2008.02015.x PMID: 18823504

27. Devaraj S, Singh U, Jialal I. Human C-reactive protein and the metabolic syndrome. Curr Opin Lipidol. 2009;20(3):182-9. DOI: 10.1097/MOL.0b013e32832ac03e PMID: 19369869

28. Libby P, Ridker PM. Novel inflammatory markers of coronary risk theory versus practice. Circ. 1999;100(11):1148-50.

29. Rietzschel E, De Buyzere M. High-sensitive C-reactive protein: universal prognostic and causative biomarker in heart disease? Biomark Med. 2012;6(1):19-34. DOI: 10.2217/bmm.11.108 PMID: 22296194

30. Xu YL, Li JJ, Xu B, Zhu CG, Yang YJ, Chen JL, et al. Increased plasma $\mathrm{C}$-reactive protein level predicts rapid progression of non-target atherosclerotic lesions in patients with stable angina after stenting. Chin Med J (Engl). 2011;124(19):3022-9. PMID: 22040548

31. He P, Xie X, Ding Y, Chen X. [Correlation between high sensitive C-reactive protein, lipoprotein(a), blood uric acid and severity of coronary artery disease]. Zhonghua. 2010;90(28):1989-91. 